\title{
The strain softening model of rock damage under compression and tension
}

\author{
Xiaoen Wu $\mathbf{u}^{(1,2)}$, Pingyuan Yang(3), Junhua Chen ${ }^{(4)}$ \\ (1) Business School, Central South University, Changsha 410083, CHINA \\ (2) China Energy Engineering Group Equipment Co. Ltd., Beijing 100044, CHINA \\ (3) China Power Construction Engineering Consulting Co. Ltd., Beijing 100011, CHINA \\ e-mail: pingyuanyang2008@163.com \\ (4) School of Civil Engineering, Central South University, Changsha 410075, CHINA
}

\section{SUMMARY}

Deformation and failure of brittle material under compression are different from those under tension. The differences are characteristic for the brittle material such as rock, concrete and others. At present, few constitutive models for rock can reflect the differences. A damage-induced softening model for rock constitutive relations is presented based on statistical strength theory, continuum damage mechanics and elastic mechanics. The model can consider properties of rock mechanics such as strain softening, difference in strength between compression and tension, nonliner stress-strain relation, compressive hardening, brittleness and so on. The model is welladapted, simple and practical as it is flexible and has only 7 parameters which can be easily obtained from uniaxial test under compression and tension. Under triaxial compression, uniaxial compression and uniaxial tension, the stress-strain relations obtained from the presented model are compared with those obtained from laboratory tests. The comparisons show that the differences between results obtained respectively from the presented model and laboratory tests are small. The presented model is rational.

KEY WORDS: $\quad$ rock mechanics; damage mechanics; constitutive relation; compression; tension; strain softening.

\section{INTRODUCTION}

Rock is initially damaged material with holes, cracks and other defects. The non-linearity of brittleness, stress-strain relationship, strain softening, compressive rigidity and tensile strength makes the rock different from mechanical properties of homogeneous materials such as metals. At present, elastic-plastic mechanics theory, fracture mechanics theory and damage mechanics theory are three main theories used to study the mechanical properties of rock. The classical elastic-plastic theory neglects the non-linearity of the stress-strain relationship of rock and can't reflect the brittleness of rock breaking. Since the 1950s, fracture mechanics 
theory has developed on the basis of classical elastic-plastic theory. Fracture mechanics theory recognizes the existence of defects in the form of cracks in rock materials, and holds that the existence of defects leads to the strength of the rock material being lower than the strength predicted by classical elastic mechanics theory or the strength of non-defective rock. This theory focuses on rock breaking. In particular, the elastic-fracture mechanics theory usually assumes that the stress-strain before the peak value is linear elasticity. When the stress reaches the peak value, the rock cracks and the stress falls to 0 . The fracture mechanics theory neglects the strain softening of rock after stress peak. Since the 1970s, continuum damage mechanics has gradually become the main theory to study the mechanical properties of brittle materials such as concrete and rock. Compared with classical elastic-plastic mechanics and fracture mechanics theory, damage mechanics boasts great advantages in explaining rock brittleness, non-linearity of stress-strain relationship, strain softening, unequal compressive and tensile strength and compressive rigidity. Rock damage constitutive model is not only the focus of research, but also the one most extensively used in engineering.

The commonly used rock damage constitutive model is continuum damage model, which mainly includes macroscopic and microscopic damage model [1-7]. The former establishes damage evolution equation based on macroscopic phenomenon or test result of laboratory test. Then it combines the damage evolution relation and classical elastic constitutive theory with fracture mechanics constitutive theory through assumption. The latter assumes that the macroscopic element is composed of microscopic element. It finds the relationship between the change law of defects and the stress-strain relation of the element from the microscopic point of view, and obtains the damage evolution relation. Then it is all related to the classical elastic constitutive theory and fracture mechanics constitutive theory so that the damage constitutive relation is obtained. Considering the mechanical mechanism of damage, the microscopic damage model is more complex than the macroscopic damage model but its applicability is substantial than that of the macroscopic damage model.

The common rock damage models include both compression and tension damage model. Grady and Kipp [8], Taylor et al. [9-10], Kuszmaul [11], Liu and Katsabanisj [12] held that volumetric tensile stress can cause crack propagation in rock and damage to its mechanical properties. Based on this, a microscopic volume tension damage model has been established. These models are elastic damage models that don't consider rock damage under volumetric compressive stress. Therefore, according to these models, the uniaxial or triaxial tension damage constitutive relation of rock can be obtained, but the uniaxial or triaxial compression damage constitutive relation can't. Cao Wengui et al., Xu Weiya and Wei Lide, Wei Lide et al., Zeng Sheng et al. established the microscopic-statistical elastic damage model under volumetric compressive stress according to the strength statistical theory. In addition, according to these models, the constitutive relation of uniaxial or triaxial compression damage of rock can be obtained, but the constitutive relation of uniaxial or triaxial tension can't. According to Yang et al., the mechanical damage caused by crack propagation in rock under both tension and compression is deteriorated. Based on the different compressive strength and tensile strength of rock, the microscopic equivalent tension damage model has been established. The model is a damage model under compression and tension, which can reflect the constitutive relation of rock damage when the volumetric stress is compressive or tensile. However, the ratio between the uniaxial tensile strength and the uniaxial compressive strength of the rock calculated from this model is twice of Poisson's ratio. The ratio is large, greatly different from the test result. Therefore, the damage model proposed by Yang et al. [13] fails to reflect correctly the difference of compressive and tensile strength of rock. 
In view of the shortcomings of the damage models mentioned above, this study proposes a constitutive model of elastic microscopic damage considering the softening effect of compressive and tensile damage according to the strength statistical theory and elastic mechanics theory, and verifies the rationality of the proposed model by triaxial compression test and uniaxial tension test, hoping that the proposed model can reflect well the brittleness, non-linearity of stress-strain relationship, strain softening, unequal compressive and tensile strength and compression rigidity of rock so as to provide reference for the study of the rock damage model.

\section{CONSTITUTIVE RELATION OF ROCK DAMAGE UNDER COMPRESSION AND TENSION}

\subsection{MICROSCOPIC DAMAGE EVOLUTION LAW}

(1) Microscopic damage probability density model

According to the classical damage model, the main breaking mode of rock includes elastic brittle breaking caused by the damage accumulation. The damage evolution of microscopic element is generally determined by the variation of crack density in rock elements. Assuming that the rock is an isotropic material, the general expression of the damage variable is:

$$
D=f\left(C_{d}\right)
$$

Where, $D$ is the damage variable of rock element, which is scalar, $C_{d}$ is the crack density activated by loading, which is the crack number of unit volume; $f$ is an expression with the crack density as a variable.

The higher the crack density activated by microscopic element is, the greater the probability of breaking is. The existing researches show that in laboratory test, the strength of rock specimen obeys normal distribution, Weibull distribution and exponential distribution. From the microscopic point of view, when the element reaches the strength, that is, when the element is broken, the activated crack density reaches the critical value. The higher the strength is, the cracks are more activated. Therefore, when the intensity of the element is characterized by the crack density activated in the element, it also obeys the statistical law. In this study, it is assumed that the breaking probability of microscopic element with volume $V_{0}$ obeys Weibull distribution, and the breaking probability is:

$$
\begin{gathered}
p_{f}=1-e^{-A N_{d}^{B}} \\
N_{d}=V_{0} C_{d}
\end{gathered}
$$

Where, $A$ and $B$ are the parameters of Weibull distribution function; $N_{d}$ is the number of activated cracks. $V_{0}$ is the volume of microscopic element.

The damage degree of rock is expressed by the breaking probability, and the damage value of microscopic element is calculated as:

$$
D=p_{f}=1-e^{-A\left(V_{0} C_{d}\right)^{B}}
$$

$C_{d}$ is determined either by the strain or stress level: 


$$
C_{d}=F_{1}\left(\sigma_{i j}\right) \text { or } C_{d}=F_{2}\left(\varepsilon_{i j}\right)
$$

Where, $F_{1}$ and $F_{2}$ are crack density functions with stress and strain as variables respectively. $\sigma_{i j}$ and $\varepsilon_{i j}$ are the stress tensor component and the strain tensor component respectively.

(2) Damage evolution equation

Grady and Kipp, Taylor et al. held that the crack density will be activated only under the effect of volumetric tension or volumetric tensile stress. Their proposed damage model isn't applicable to rocks subjected to triaxial compressive stress. Chen et al. and Yang et al. believed that damage grows or crack density increases as long as tensile deformation occurs in the element. The tensile deformation of the element doesn't necessarily lead to volumetric tension, but volumetric tension must be accompanied by the tensile deformation. Thus, the generation of damage is not necessarily accompanied by the volumetric tension. They put forward the concept of equivalent tensile deformation. Compared with Grady and Kipp, Taylor et al., Chen et al. and Yang et al. moderated the conditions for damage generation.

The relationship between the activated crack density and the equivalent tensile deformation is:

$$
\begin{gathered}
C_{d}=F_{2}\left(\varepsilon_{i j}\right)=\alpha\left(\theta-\theta_{c r}\right)^{\beta} \\
\theta=\sum_{i=1}^{3}\left[\left(\varepsilon_{i}+\left|\varepsilon_{i}\right|\right) / 2\right]
\end{gathered}
$$

Where, $\theta$ is equivalent tensile deformation; $\theta_{c r}$ is the equivalent tensile deformation threshold; $\varepsilon_{i}$ is the principal strain of the direction, $i=1$, 2, 3. In this study, the mechanical positive and negative numbers are as follows: compression is negative, tension is positive. $\alpha$ and $\beta$ are material parameters.

According to Eq. (4), the damage evolution equation is:

$$
D=1-e^{-A\left(V_{0} \alpha\right)^{B}\left(\theta-\theta_{c r}\right)^{\beta \cdot B}}=1-e^{-m\left(\theta-\theta_{c r}\right)^{n}}
$$

where $m$ and $n$ are material parameters, $m=A\left(V_{0} \alpha\right)^{B}, n=\beta \cdot B$.

The existing researches show that the deterioration rate of mechanical properties of rock under volumetric compressive and tensile stress is different because there is significant friction effect and residual strength in rock under relative volumetric tensile and volumetric compressive stress. Therefore, with the increase of equivalent tensile strain, the rate of damage growth is different between the volumetric compressive stress and the volumetric tensile stress. Thus, under volumetric compressive stress and under volumetric tensile stress the two Weibull distribution parameters of the damage evolution Eq. (8) are different which leads to the difference of the parameters $m$ and $n$ of the damage evolution Eq. (8) under volumetric compressive stress and the parameters $m$ and $n$ under volumetric tensile stress.

For the elastic damage relation, the volumetric compressive stress and the volumetric tensile stress are equivalent to the volumetric compressive deformation and the volumetric tensile deformation respectively. Therefore, the damage evolution Eq. (8) needs to be corrected. After correction, the damage evolution equation is:

$$
D= \begin{cases}1-e^{-m_{t}\left(\theta-\theta_{c r}\right)^{n_{t}}}, & \varepsilon_{v} \geq 0 \\ 1-e^{-m_{c}\left(\theta-\theta_{c r}\right)^{n_{c}}}, & \varepsilon_{v}<0\end{cases}
$$




$$
\varepsilon_{v}=\sum_{i=1}^{3} \varepsilon_{i}
$$

where, $\varepsilon_{v}$ is volumetric strain: $>0$ represents tension and $<0$ represents compression; $m_{t}$ and $n_{t}$ are material parameters under the volumetric tensile damage. $m_{c}$ and $n_{c}$ are material parameters under the volumetric compressive damage.

With the establishment of Eq. (9) it is conciliated that the damage growth rate under volumetric compression is smaller than that under volumetric tension. Then:

$$
\left.\frac{d D}{d \theta}\right|_{\varepsilon_{v} \geq 0}>\left.\frac{d D}{d \theta}\right|_{\varepsilon_{v}<0}
$$

\subsection{DAMAGE CONSTITUTIVE RELATION}

(1) Constitutive relation of elastic damage

When the damage occurs, there is elastic damage change in the rock. The increase of damage will lead to the decrease of bulk modulus, shear modulus and elastic modulus of rock. The relationship between the damage modulus and the undamaged modulus is:

$$
\begin{aligned}
& K=\bar{K}(1-D) \\
& G=\bar{G}(1-D) \\
& E=\bar{E}(1-D)
\end{aligned}
$$

In the Eqs. (12)-(13), $\bar{K}, \bar{G}$ and $\bar{E}$ are respectively the bulk modulus, shear modulus and elastic modulus of the undamaged rock; $K, G$ and $E$ are the bulk modulus and shear modulus of the damage rock respectively.

The Poisson's ratio is small and the effect of Poisson's ratio variation on the model calculation results is also small, so it is generally assumed that damage doesn't affect Poisson's ratio. Thus, there are the following relationship equations:

$$
\begin{gathered}
\mu=\bar{\mu} \\
E=2 G(1+\mu)=3 K(1+\mu) \\
\bar{E}=2 \bar{G}(1+\bar{\mu})=3 \bar{K}(1+\bar{\mu})
\end{gathered}
$$

Where, $\bar{\mu}$ and $\mu$ are undamaged Poisson's ratio and damage Poisson's ratio.

If the stress is decomposed into spherical and deviatorical part, the constitutive relation of rock elastic damage is as follows:

$$
\begin{gathered}
\sigma_{m}=\left(\sigma_{1}+\sigma_{2}+\sigma_{3}\right) / 3=K \varepsilon_{v}^{e}=\bar{K}(1-D) \varepsilon_{v} \\
s_{i j}=2 G e_{i j}^{e}=2 \bar{G}(1-D) e_{i j}
\end{gathered}
$$

where, $\sigma_{m}$ is the spherical stress component, $\sigma_{1}, \sigma_{2}$ and $\sigma_{3}$ are the maximum principal stress, the intermediate principal stress and the minimum principal stress, respectively. $\sigma_{1} \geq \sigma_{2} \geq \sigma_{3} ; s_{i j}$ is the component of the deviatorical stress tensor; $\varepsilon_{v}^{e}$ and $e_{i j}^{e}$ are elastic 
volumetric deformation and elastic partial strain with $i, j=1,2$, 3. For the elastic damage relationship, $\varepsilon_{v}^{e}=\varepsilon_{v}, e_{i j}^{e}=e_{i j}$.

The damage evolution Eq. (9) and the constitutive relation Eqs. (18) and (19) comprise the constitutive relation of elastic damage under volumetric compression and tension.

\subsection{MEANS FOR OBTAINING PARAMETERS}

(1) Determining $\varepsilon_{t}^{e}, \bar{E}$ and $\bar{\mu}$

The parameters of the damage model in this study can be determined by laboratory static compression or static tensile test. From Eqs. (8)-(19), it can be seen that for the rock sample of a certain volume, the parameters required for the damage constitutive relation calculation are only 7, including $m_{t}, n_{t}, m_{c}, n_{c}, \varepsilon_{t}^{e}, \bar{\mu}$ and $\bar{E}$. According to Eq. (16) and Eq. (17), $\bar{\mu}$ and $\bar{E}$ may also be substituted by $\bar{K}$ and $\bar{G}$.

Under uniaxial static loading, the equivalent tensile strain threshold $\theta_{c r}$ is calculated as:

$$
\theta_{c r}=\varepsilon_{t}^{e}=\frac{\sigma_{t}^{e}}{\bar{E}}=-2 \bar{\mu} \varepsilon_{c}^{e}=-2 \bar{\mu} \frac{\sigma_{c}^{e}}{E_{0}}
$$

Where, $\sigma_{t}^{e}$ and $\sigma_{c}^{e}$ are linear elastic ultimate stress under uniaxial tension and uniaxial compression, respectively; $\varepsilon_{c}^{e}$ and $\varepsilon_{t}^{e}$ are linear elastic limit strain values under uniaxial static compression and uniaxial static tension respectively. Negative sign means that the compression is negative and the tension is positive.

Since the compression test is simpler than the tensile test, the linear elastic limit stress $\sigma_{c}^{e}$, the linear elastic limit strain $\varepsilon_{c}^{e}$, the undamaged elastic modulus $\bar{E}$ and the undamaged Poisson's ratio $\bar{\mu}$ can be determined by the laboratory uniaxial static compression test, and then $\varepsilon_{t}^{e}$ or $\theta_{c r}$ is determined by the Eq. (20).

(2) Determining $m_{t}$ and $n_{t}, m_{c}$ and $n_{c}$

The damage variable ranges from 0 to 1 . For rock element, $D=0$ corresponds to an undamaged state and $D=1$ corresponds to fully broken state. For the laboratory triaxial test, the damage variable increases with loading. When the damage variable reaches a certain value between 0 and 1, the rock element begins to get damaged seriously, and the stress of the element reaches the peak strength. At this time, the damage variable satisfies the following equation:

$$
\begin{aligned}
& D_{t-\lim }=1-e^{-m_{t}\left(\theta_{\text {lim }}-\theta_{c r}\right)^{n_{t}}} \\
& D_{c-\lim }=1-e^{-m_{c}\left(\theta_{\text {lim }}-\theta_{c r}\right)^{n_{c}}}
\end{aligned}
$$

Where, $D_{t \text {-lim }}$ and $D_{c \text {-lim }}$ are the critical damage variables under volumetric tension and volumetric compression respectively. $\theta_{t \text {-lim }}$ and $\theta_{c \text {-lim }}$ are the critical equivalent tensile strain under volumetric tension and volumetric compression respectively. $D_{t \text {-lim }}, D_{c \text {-lim }}, D_{\text {lim }}$ and $\theta_{\text {lim }}$ correspond to the peak stress state. 
According to the Eqs. (12)-(19), the uniaxial compressive strength and the uniaxial tensile strength satisfy:

$$
\begin{gathered}
\sigma_{c}=\bar{E} \varepsilon_{c}\left(1-D_{c-l i m}\right)=E_{c-l i m} \varepsilon_{c}=\bar{E} \varepsilon_{c} e^{-m\left(-2 k \mu \varepsilon_{c}-\theta_{c r}\right)^{n}} \\
\sigma_{t}=\bar{E} \varepsilon_{t}\left(1-D_{t-\lim }\right)=E_{t-l i m} \varepsilon_{t}=\bar{E} \varepsilon_{t} e^{-m\left(\varepsilon_{t}-\theta_{c r}\right)^{n}}
\end{gathered}
$$

Where, $\sigma_{c}$ and $\sigma_{t}$ are uniaxial compressive strength and uniaxial tensile strength respectively. $E_{t \text {-lim }}$ and $E_{c \text {-lim }}$ are the ratios of stress and strain at the stress peak point in uniaxial tensile and compressive tests respectively, $E_{t \text {-lim }}=\bar{E}\left(1-D_{t-\lim }\right), E_{c-\lim }=\bar{E}\left(1-D_{c-\lim }\right)$. $\varepsilon_{c}$ and $\varepsilon_{t}$ are the strains corresponding to the peak stress in uniaxial static compression test and tensile test respectively.

The existing researches show that $D_{t \text {-lim }}=0.1 \sim 0.2, D_{c \text {-lim }}=0.2 \sim 0.5$. The brittleness under volumetric tension is more significant than that under volumetric compression, so $D_{\text {t-lim }} \leq D_{\text {-lim }} D_{t \text {-lim }} \leq D_{\text {-lim }}$.

Under uniaxial compression, $\sigma_{3}<0, \sigma_{1}=\sigma_{2}=0$, the tangent modulus of elasticity at the stress peak point is equal to 0 , and the following equation holds:

$$
\left.\frac{d \sigma_{3}}{d \varepsilon_{3}}\right|_{\varepsilon_{3}=\varepsilon_{c}}=\frac{\bar{E}\left[1+2 k \bar{\mu} m_{c} n_{c} \varepsilon_{3}\left(-2 k \bar{\mu} \varepsilon_{3}-\theta_{c r}\right)^{n_{c}-1}\right]}{e^{m_{c}\left(-2 \bar{\mu} \varepsilon_{3}-\theta_{c r}\right)^{c_{c}}}}=0
$$

Under uniaxial tensile condition $\sigma_{1}<0, \sigma_{3}=\sigma_{3}=0$, the tangent modulus of elasticity at the stress peak point is equal to 0 , and the following equation stands:

$$
\left.\frac{d \sigma_{1}}{d \varepsilon_{1}}\right|_{\varepsilon_{1}=\varepsilon_{t}}=\frac{\bar{E}\left[1+m_{t} n_{t} \varepsilon_{1}\left(\varepsilon_{1}-\theta_{c r}\right)^{n_{t}-1}\right]}{e^{m_{t}\left(\varepsilon_{1}-\theta_{c r}\right)^{n_{t}}}}=0
$$

Combining the Eqs. (23) and (25), (24) and (26), $m_{t}$ and $n_{t}, m_{c}$ and $n_{c}$ can be calculated.

\section{MODEL VERIFICATION}

\subsection{MEANS FOR VERIFICATION}

The stress-strain relationship of rock is very complex, which is related to the loading condition. The stress-strain relationship is relatively simple under the laboratory routine test condition, so the constitutive model is usually verified by the laboratory routine test result. Cao Wengui et al., Wei Lide et al. and Zeng Sheng et al. all adopt this method to verify the constitutive model. For the model validation in this study, the test parameters and test conditions are following: the colour of rock is gray-dark gray, dense limestone, belonging to the rock sample $\bar{E}=40.7 \mathrm{GPa}, \bar{\mu}=0.30, \theta_{c r}=2.0 \times 10^{-4}, m_{c}=8.37 \times 10^{8}, n_{c}=3.76, m_{t}=1.51 \times 10^{9}, n_{t}=2.72$. The uniaxial compressive strength is $-165.3 \mathrm{MPa}$ and the uniaxial tensile strength is 14.4 MPa. The axial loading strain rate is $5 \times 10^{-5} / \mathrm{s}^{-1}$. The relationship between stress and strain under triaxial compression test, uniaxial compressive test and uniaxial tensile test is given in the literature. 


\subsection{RESULTS AND ANALYSIS}

The axial stress-strain curves under triaxial or uniaxial loading are calculated according to the parameters given in the experiment and the constitutive relation proposed in this study. The axial stress and strain curve under compressive conditions is shown in Figure 1. The uniaxial tensile stress and strain curve is shown in Figure 2.

The calculated stress-strain curve has the following main characteristics:

(1) As can be seen from Figure 1 and Figure 2, the stress-strain curve first experiences a linear growth stage under both compressive and tensile conditions. When the elastic proportional limit is exceeded, the stress-strain relationship is in a nonlinear growth stage, but the growth stage is slower than the linear elastic stage. As the strain increases, the strain softening phenomenon occurs when the stress reaches the peak value. These properties are consistent with rock properties.

(2) Figure 1 shows that the axial peak stress or compressive strength of rock increases with the increase of confining pressure, indicating that the constitutive model can reflect the compressive rigidity of rock.

(3) By comparing Figure 1 and Figure 2, it can be seen that the uniaxial compressive strength of the rock is about 11 times of the uniaxial tensile strength, namely, the compressive strength of the rock is much greater than the tensile strength, which is also in accordance with the characteristics of the rock.

As can be seen from Figure 1 and Figure 2, for the test results and the calculation results, the curve before stress reaches the peak is well coordinated, and is relatively weak after the peak. The curve obtained from the test is consistent with that obtained from the damage model. The reason for the difference between the test result and the calculation result is that the rock is not completely elastomer, and the strain of the rock sample should include elastic strain and plastic strain, that is, the rock will produce irreversible plastic deformation along with elastic deformation. The constitutive model proposed in this study is elastic damage model. For the purpose of facilitating the calculation and application, the plastic deformation is neglected. Before the stress reaches the peak value, the plastic strain produced by the rock is small, so the plastic strain can be ignored. And the difference between the curve obtained from the test and the curve obtained from the elastic damage model is small. When the stress reaches the peak value, the plastic strain increases with the increase of deformation, and the difference between the curve obtained from the test and the curve obtained from the elastic damage model is large. The elastic brittleness of rock is generally more significant than plasticity, so even if there is a gap, it is not too large. On the whole, the shape and variation of the curve obtained from the test and the curve obtained from the damage model are consistent, and the degree of coincidence of the curve is also high. Therefore, although the damage model in this study is simple, it is reasonable. 


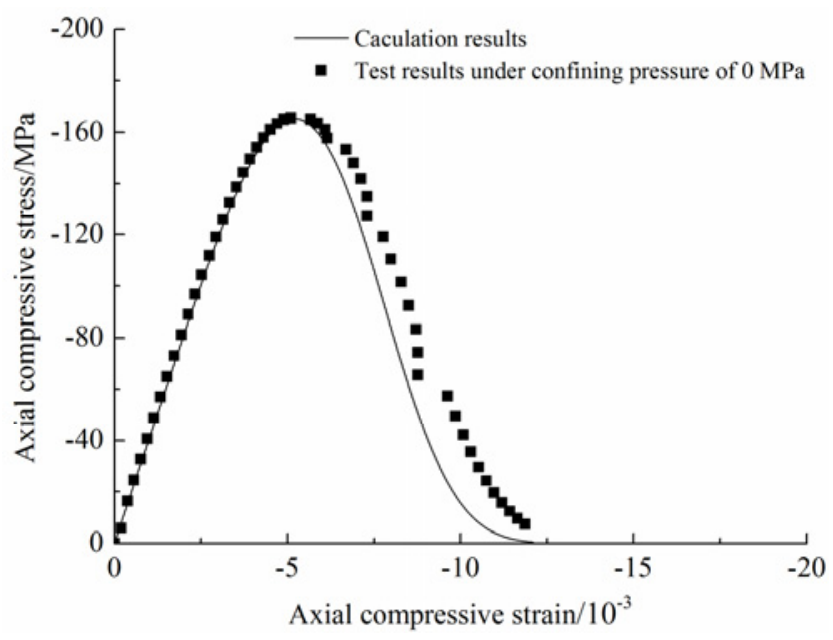

(a) Confining pressure is OMPa_(uniaxial compression)

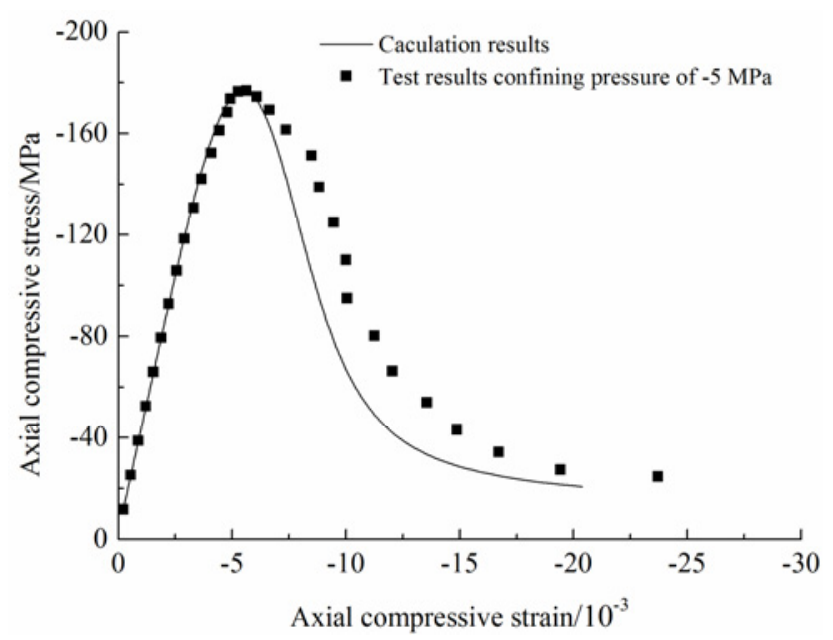

(b) Confining pressure is $-5 \mathrm{MPa}$

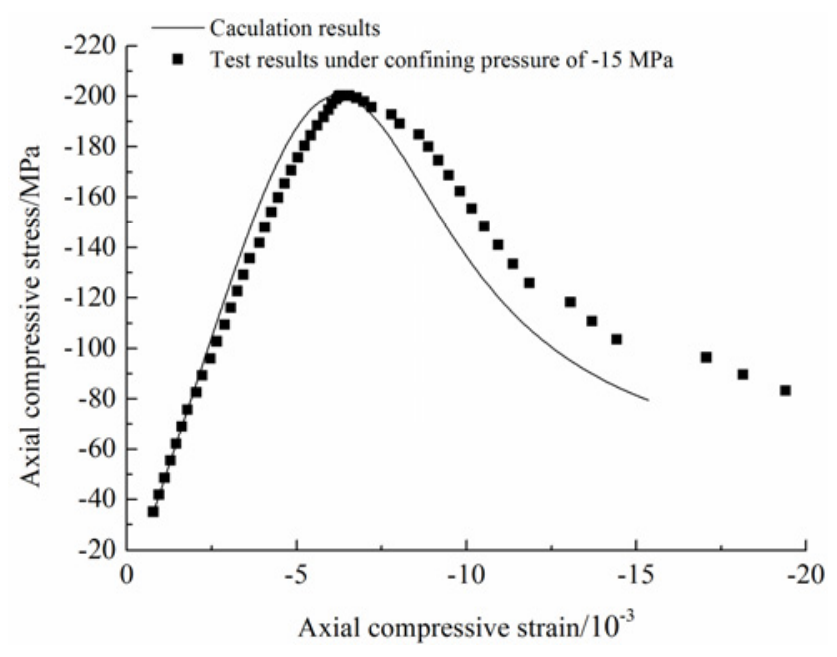

(c) Confining pressure is $-15 \mathrm{MPa}$

Fig. 1 Triaxial compression test result 


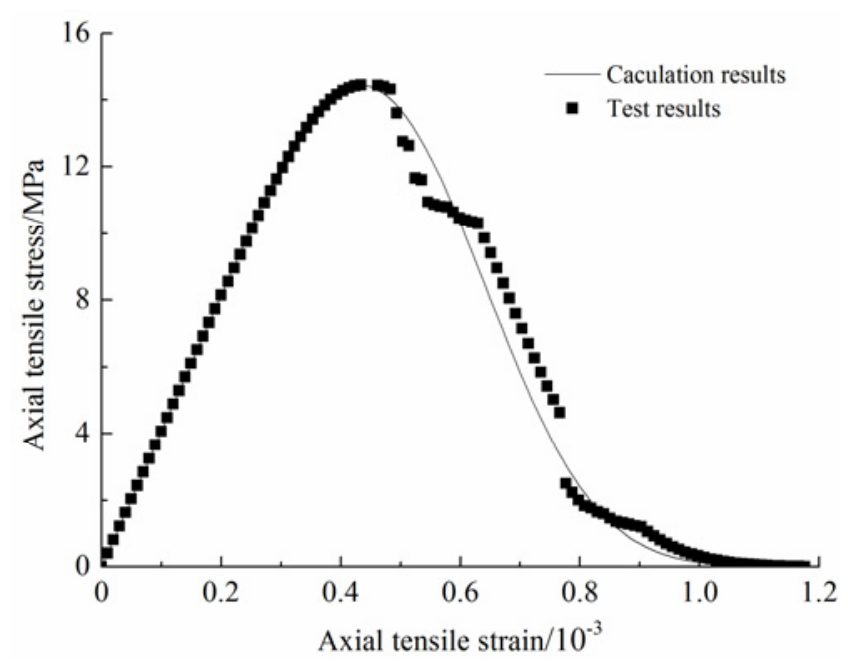

Fig. 2 Uniaxial tensile test result

\section{CONCLUSIONS}

(1) According to the strength statistical theory and the different characteristics of compressive and tensile strength of rock, the microscopic damage evolution model is proposed, and then the elastic statistical damage model is obtained combining the linear elastic mechanics theory.

(2) In the proposed model, the damage evolution relationship is divided into volumetric compression and volumetric tension damage. The model can fully reflect characteristics of rock, such as rock compression, tensile strength, strain softening, nonlinear stress-strain relationship, compressive rigidity, brittleness and so on.

(3) According to the proposed damage model under compression and tension, both the conventional triaxial compressive and uniaxial tensile stress-strain curves are obtained. The results of laboratory test are compared with the calculated results, showing that the proposed damage model under compression and tension is reasonable.

(4) There are a few calculation parameters of the proposed damage model under compression, so the calculation parameters can be easily obtained by uniaxial tension and compression tests. As a whole, the proposed model is simple and practical with a certain physical mechanism background, strong theory, and wide adaptability.

\section{REFERENCES}

[1] A.P. Jivkov, Structure of micro-crack population and damage evolution in quasi-brittle media, Theoretical \& Applied Fracture Mechanics, Vol. 70, pp. 1-9, 2014.

https://doi.org/10.1016/j.tafmec.2014.04.003

[2] S. Levasseur, F. Collin and R. Charlier, On micromechanical damage modelling in geomechanics: Influence of numerical integration scheme, Journal of Computational \& Applied Mathematics, Vol. 246, pp. 215-224, 2013.

https://doi.org/10.1016/j.cam.2012.05.022 
[3] A. Shojaei, A.D. Taleghani and G. Li, A continuum damage failure model for hydraulic fracturing of porous rocks, International Journal of Plasticity, Vol. 59, pp. 199-212, 2014. https://doi.org/10.1016/j.ijplas.2014.03.003

[4] Q. Zhu and J. Shao, Micromechanics of rock damage: Advances in the quasi-brittle field, Journal of Rock Mechanics and Geotechnical Engineering, Vol. 9, No. 1, pp. 29-40, 2017. https://doi.org/10.1016/j.jrmge.2016.11.003

[5] L.A. Nazarov, L.A. Nazarova and P.A. Tsoi, Modelling evolution of damage in rock specimens under loading, Journal of Mining Science, Vol. 51, No. 6, pp. 1101-1107, 2015. https://doi.org/10.1134/S1062739115060356

[6] S. Souissi, E. Hamdi and H. Sellami, Microstructure Effect on Hard Rock Damage and Fracture During Indentation Process, Geotechnical \& Geological Engineering, Vo. 33, No. 6, pp. 1539-1550, 2015. https://doi.org/10.1007/s10706-015-9920-6

[7] H.Y. Liu and T.M. Su, A dynamic damage constitutive model for a rock mass with nonpersistent joints under uniaxial compression, Chinese Journal of Geotechnical Engineering, Vol. 77, pp. 12-20, 2016.

https://doi.org/10.1016/j.mechrescom.2016.08.006

[8] D.E. Grady and M.E. Kipp, Continuum Modeling of Explosive Fracture in Oil Shale, International Journal of Rock Mechanics \& Mining Science \& Geomechanics Abstracts, Vol. 17, No. 3, pp. 147-157, 1980. https://doi.org/10.1016/0148-9062(80)91361-3

[9] L.M. Taylor, E.P. Chen and J.S. Kuszmaul, Microcrack-induced damage accumulation in brittle rock under dynamic loading, Computer Methods in Applied Mechanics and Enginering, Vol. 55, No. 3, pp. 301-320, 1986.

https://doi.org/10.1016/0045-7825(86)90057-5

[10] L.M. Taylor, J.S. Kuszmaul and E.P. Ghen, Damage accumulation due to macrocracking in brittle rock under dynamic loading, American Society of Mechanical Engineers, Vol. 69, pp. 95-104, 1985.

[11] J.S. Kuszmaul, A New Constitution Model for Fragmentation of Rock under Dynamic Loading, Proceedings of the 2nd International Symposium on Rock Fragmentation by Blasting, pp. 412-423, 1987.

[12] L.Q. Liu and P.D. Katsabanis, Development of a continuum damage model for blasting analysis, International Journal of Rock Mechanics and Mining Sciences, Vol. 34, No. 2, pp. 217-231, 1997. https://doi.org/10.1016/S0148-9062(96)00041-1

[13] R. Yang, W.F. Brwden and P.D. Katsabanis, A new constitutive model for blast damage, International Journal of Rock Mechanics and Mining Sciences, Vol. 33, No. 3, pp. 245-254, 1996. https://doi.org/10.1016/0148-9062(95)00064-X 